European Organization For Particle Physics Research

CERN - TIS - TE / IR / 99-08

\title{
Behaviour of Organic Materials in Radiation Environment
}

\author{
Marc Tavlet and Sorin Ilie \\ CERN, TIS and EST Divisions \\ 1211 Geneva 23, Switzerland
}

\begin{abstract}
Keywords: radiation, dose-rate, ageing, polymer, organic, insulator

Radiation effects in polymers are reminded together with the ageing factors. Radiation ageing results are mainly discussed about thermosetting insulators, structural composites and cable-insulating materials. Some hints are also given about high-voltage insulations, cooling fluids, organic scintillators and lightguides. Some parameters to be taken into account for the estimate of the lifetime of components in radiation environment are also shown.
\end{abstract}

Communication presented at the RADECS ' $99,5^{\text {th }}$ European Conference on RADtion and its Effects on Components and Systems Fontevraud (Fr), September $13-17,1999$ 


\title{
Behaviour of Organic Materials in Radiation Environment
}

\author{
Marc Tavlet and Sorin Ilie \\ CERN, TIS and EST Divisions \\ 1211 Geneva 23, Switzerland \\ tel: +41227673717 - e-mail: marc.tavlet@cern.ch
}

Keywords: radiation, dose-rate, ageing, polymer, organic, insulator

\begin{abstract}
Radiation effects in polymers are reminded, together with the ageing factors. Radiation-ageing results are mainly discussed about thermosetting insulators, structural composites and cable-insulating materials. Some hints are also given about high-voltage insulations, cooling fluids, organic scintillators and light-guides. Some parameters to be taken into account for the estimate of the lifetime of components in radiation environment are also shown.
\end{abstract}

\section{INTRODUCTION}

At the European Organization for Particle Physics Research (CERN) several high-energy particle accelerators and storage rings have been in operation for more than 40 years. The next one will be the Large Hadron Collider (LHC) located in the same $27 \mathrm{~km}$ circular tunnel as the Large Electron-Positron Collider (LEP). Its superconducting magnets will operate at liquid-helium temperature and will enable the acceleration of protons up to the energy of $7 \mathrm{TeV}$; it will be the largest and the most powerful particle accelerator in the world. Due to beam losses in proton machines and due to synchrotron radiation in lepton machines, all materials and components installed in the tunnels are exposed to ionising radiation and radiation damage is observed [1]. From these colliders head-on collisions between primary particle beams circulating in opposite directions are provoked in associated physics detectors. From the collisions between stable particles, exotic unstable particles are produced. The study of their characteristics and behaviour allows to understand the fundamental laws of physics. With the highenergy, high-intensity collisions produced from the LHC, radiation damage is also to be expected in particle-physics detectors.

Therefore, for a safe and reliable operation, the radiation behaviour of most of the components has to be tested prior to their selection. From the very beginning, attention has been given to the effects of ionising radiation on polymeric-based components. Radiation-resistance tests are performed on a routine basis on magnet-coil insulations, on cable-insulating materials and on structural composites; mechanical tests are carried out in accordance with the standard 544 of the International Electrotechnical Commis-sion (IEC) [2]. Moreover, electrical properties of highvoltage insulations and optical properties of organic scintillators and wave-guides are also studied. For these components, transient effects have also to be taken into account. At dose rates and dose levels encountered around particle accelerators as well as in space, metal alloys and ceramics do not suffer from any radiation damage or particular ageing. Our long-term experience has pointed out many parameters to be taken into account for the estimate of the lifetime of organic components in radiation environments. A large amount of results are published in the form of catalogues for a large variety of commercially available compounds and components [3].

\section{PRIMARY RADIATION EFFECTS IN POLYMERIC ORGANIC MATERIALS}

Due to the type of physico-chemical bonds in polymers, they are more sensitive to ionising radiation than metals and ceramics. Common polymers are based on long hydrogenated carbon chains. In most polymers, some of the hydrogen atoms are substituted with side groups. The main chain and/or the side groups may be linear or cyclic, they may contain mineral atoms. If the sided groups are long chains similar to the main one, the polymer is said to be branched, and its crystallinity will usually be low. In cross-linked thermoplastics and in thermosets, the chains are linked together in a three dimensional network with low or no crystallinity. 
Whatever is the type of radiation (charged or neutral particle, or electromagnetic radiation of enough energy), its effect always results in excitation and ionisation of the polymer chain. Polymers are usually electrical insulators; if they are subjected to high dose-rate irradiation, the number of free electrons may reduce significantly their resistivity, but this is only a transient effect. If charged particles are used, electric charges may accumulate in organic insulators.

After de-excitation and capture of the free electron, chemical radicals are created ( $=$ species with unpaired electron). These can be more or less reactive, and the reaction may propagate along the chain or to a side chain. The principal reactions are chain scissions, cross-linkings and changes of side groups. Chain scission -also called degradation - is the random cutting of the main chains, which results in a decrease of the mean molecular weight. Degradation may also result from de-polymerisation or "unzipping" of the main chain which leads to products similar to the parent monomer. From cross-linking a three dimensional network is created in thermo-plastics. Cyclisation and changes of side groups lead to the formation of unsaturations and gas evolution. Polymers are also subject to phase changes; the crystalline phase of plastics may be destroyed by irradiation (this effect is important in PTFE); on the other hand, the energy brought by radiation may anneal amorphous plastics in which crystallites will be produced [4].

In translucent polymers, trapped electrons and unsaturations result in colour centres which cause yellowing and/or darkening of the material. Some of these colour centres may be annealed by temperature, by reaction of oxygen and/or by photo-bleaching.

When components are irradiated with neutrons or high-energy particles, some additives become radioactive. This is the case of brominated compounds added as flame retardant and/or stabiliser, as well as with the boron-containing glass fibres.

\section{AGEING OF ORGANIC MATERIALS}

Ageing is the irreversible change of at least one of the properties of a material submitted to its environment during its operational life; it has to be distinguished from the transient change due to a temporary external stress [5].
Ageing always results from a change in the intimate physico-chemical structure; materials evolve with time because they are not in a stable thermodynamic state. Physico-chemical modifications are due to the chemical processes listed above. Ageing factors and processes depend on the environment. When materials are used as components, they are subject to environmental conditions, and they inevitably age. As organic materials are used virtually everywhere in particle accelerators, as well as in nuclear power plants (NPPs), and serve vital functions, ageing of electrical insulators, seals and joints, lubricants and structural composites reduces design safety margins and may cause failures of both operational and safety systems [6].

In comparison with metals and ceramics, which are mainly sensitive to corrosion, polymers are more sensitive to ageing: they are more affected by external chemical factors such as oxidants (water, oxygen, ozone...) and pollutants including bacteria, and physical factors such as temperature (and temperature changes), mechanical stresses (including abrasion and fatigue), electrical and/or magnetic fields, light (mainly UV), microwaves, ultrasonic, ionising radiation, etc. [4, 7, 8, 9]. In any material, internal defects such as impurities, grains and cavities will usually facilitate the action of external factors. During the transformation process, the temperature is raised above a phase transition, and hydro-peroxydes which are potential initiators for further oxidative degradation are produced [10]. The proper choice of polymerisation and transformation processes is crucial for the further ageing properties. The mechanisms of ageing depend on the material type. In polymeric materials, additives such as organic or mineral fillers, and stabilisers such as antioxidants and UV protectors, may also be modified and/or eliminated with time; they usually change the mechanisms and the rate of ageing.

A side effect of gas evolution from irradiated polymeric compounds is that the evolved gas can be poisonous and/or corrosive; this is the case for example with $\mathrm{HCl}$ evolved from PVC. The radiolysis of the fluorocarbon used as cooling fluids results in the production of hydrofluoric acid which may corrode the tubing system. It is also to be noted that carbon dioxide which is often considered as an inert gas is not inert on a radiation point of view: its radiolysis results in the formation of atomic oxygen which is very corrosive. 


\section{MONITORING OF AGEING}

In order to understand the mechanisms of ageing, physico-chemical analysis such as differential scanning calorimetry, infra-red spectroscopy, electron paramagnetic spectroscopy, nuclear magnetic spectroscopy, molecular weight measurements, gel fraction measurements, etc. have to be carried out. For the characterisation of radio-induced effects on cooling fluids, several methods are used: UV-Vis and FT-IR spectrophotometry, gas chromatography, scanning electron microscopy, etc. To monitor their ageing, as well as the one of lubricating oils, it is usually enough to measure their optical absorption and/or their viscosity.

Usually, from the user's point of view, it must be possible to monitor the ageing by the follow-up of the evolution of one or several macroscopic properties. As materials are used as (part of) components, they have a (or more) specific function(s) requiring specific properties. It seems therefore logical to test the mechanical properties of structural materials, the electrical properties of insulators, the optical properties of optical components, and so forth. But this is not always neither the easiest nor the best way. Experience has shown that it is often possible to assess the ageing of an insulator from the degradation of its mechanical properties. The IEC 544 standard recommends measuring the degradation of insulators from mechanical tests [2]. Flexural tests are carried out on rigid insulators (thermosets, varnish, composites) and tensile tests on flexible insulators (mainly thermoplastics and rubbers used for cable insulation). The problem is that macroscopic samples have to be taken out of components, which is not always feasible. A nondestructive method using an indenter has been developed for cable-ageing monitoring [11]. Unfortunately, non-destructive and/or microsampling methods have not yet proven to be reliable, and reference baselines are still needed $[6,12]$.

\section{RESULTS OF RADIATION-AGEING TESTS - FACTORS OF AGEING}

As said in the introduction, for the selection of organic insulators, numerous radiation-resistance tests are carried out at CERN on a routine basis, in accordance with the IEC 544 standard which recommends that accelerated tests be performed after irradiations at dose rates higher than $1 \mathrm{~Gy} / \mathrm{s}$, and that more representative tests be performed after ageing conditions as close as possible to inlife conditions.
In particle accelerators, the most exposed organic components are the insulations of the magnet coils. They are usually cured glass-reinforced composites based on an aromatic epoxy-resin with specially formulated hardener and accelerator. These compounds may resist up to hundreds of megagrays. Today, it has not yet been possible to detect wether they may be subjected to "dose-rate effect" due to oxidation. Many results can be found in [3]. Fig. 1 show the results for such an epoxy-based prepreg used for the insulation of magnet coils in the LEP.

Epoxy systems are also often used as adhesives. Today, "high-performance plastics" may also be used as structural materials. Many radiation-test results about these engineering plastics can be found in $[3,13,14]$, some have been identified as being particularly sensitive to radiation, namely the PTFE (Teflon) and the acetal resins (e.g. Delrin). In space applications, atomic oxygen and high-energy cosmic rays may affect the tightness of insulating films.

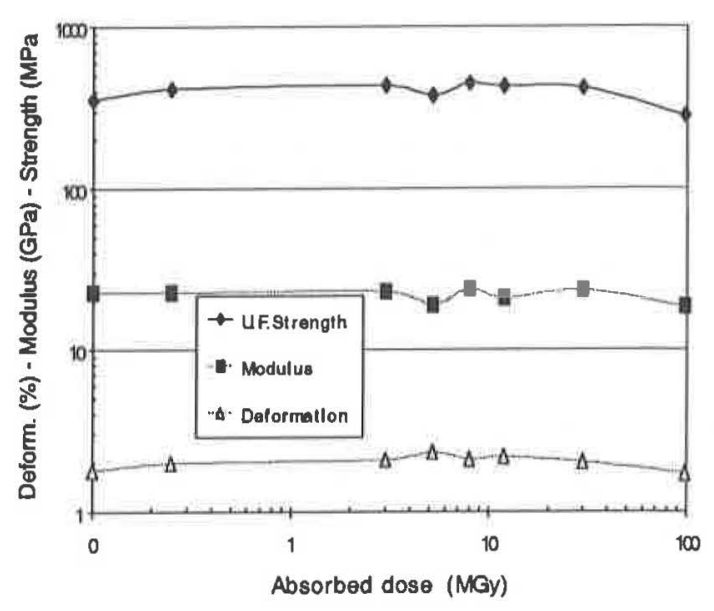

Fig. I- Results of mechanical tests showing the good radiation behaviour of a glass-fibre reinforced epoxy.

In NPPs, the more crucial organic compounds are the insulations of in-containment cables. For firesafety reasons, the current tendency is that halogenated compounds are progressively replaced by EPR/EPDM-based rubbers for power and HV cables, and by polyolefin-based compounds for instrumentation and control cables. Over the years, many of these cable insulations have been tested. The results of radiation tests have shown that polyolefin-based compounds usually present an important dose-rate effect [3, 15, 16] (see Fig.2), which may be combined with a temperature effect [17]. On the other hand, it 
appears from many results that the degradation of cable insulations does not depend on the radiation type [18].

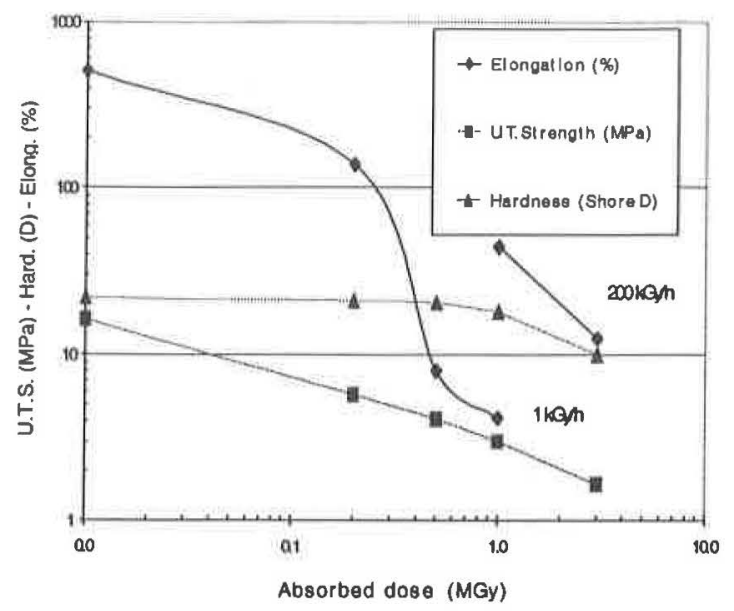

Fig. 2- Results of mechanical tests showing a strong dose-rate effect on a polyolefin cable insulation.

Tests of insulating and structural materials after irradiation at cryogenic temperature have shown that there is no significant influence of the irradiation temperature on the radiation degradation of thermosets and composites [19], while the degradation of plastic films is even less severe as they are protected against oxidation [20]. Fig. 3 compares the degradation of the mechanical properties of Kapton film irradiated either at ambient conditions or at $4 \mathrm{~K}$ in liquid helium.

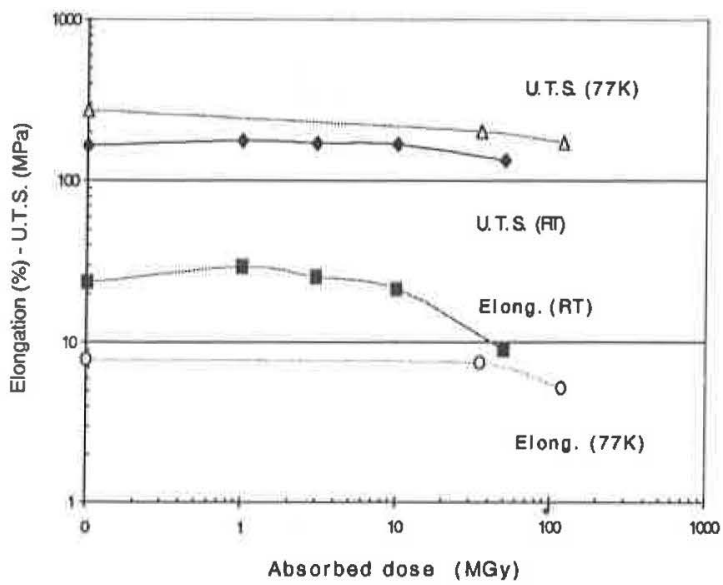

Fig. 3- Degradation of the mechanical properties of a Kapton film; comparison between irradiation at ambient conditions and at $4 \mathrm{~K}$ in liquid helium
Some experiments about the synergy between irradiation and mechanical stress have shown that rubbers and composites under stress are more sensitive to radiation and degrade faster [21]. This effect is important for rubber seals, but is negligible for structural composites used below the yield strength.

The table hereafter summarises the limit doses (for irradiation carried out in air at dose rates above 1 Gy/s) for some materials tested at CERN with regards to their mechanical properties :

Materials

Dose limits in air

Specially formulated aromatic epoxy composites 300 MGy

Cyanate ester resins 300 MGy

Poly-ether-imide (PEI) resins 50 MGy

PEI as wire insulation (Siltem) 3 MGy

Specially formulated EPR/EPDM insulated cables

Specially formulated EVA insulated cables

Usual polyolefin insulated cables

0.1 MGy

Polyimide (Kapton $H$ ) films and wire insulation 50 MGy

PEEK (amorphous) films and wire insulation

Polyarylate (Isaryl) films

10 MGy

PETP (Mylar) films 0.5 MGy

Polypropylene films $30 \mathrm{kGy}$

Polyamide 66 (Nylon) hoses or pieces 300 kGy

Acetal resins (POM) (Delrin) hoses or pieces

$30 \mathrm{kGy}$

FEP insulated wires $30 \mathrm{kGy}$

PTFE insulated wires

In inert atmosphere (in vacuum or in cryogenic liquids), the dose limits can be 3 to 10 times higher than the ones given above. The factor is higher for materials which are sensitive to oxidation, such as the polyolefins, as well as for PEEK, Nylon and PTFE. 
Experience has shown that the Young's modulus of a rigid material usually varies much less with irradiation than the ultimate flexural strength of the ultimate deformation. Polyamide 4.6 is an exception to this statement; polyamides (Nylons) are sensitive to moisture and oxidation. Fig. 4 (in which the curves correspond to irradiation in a nuclear reactor at dose rates of $200 \mathrm{kGy} / \mathrm{h}$ ) shows that the modulus of PA 4.6 is sensitive to radiation; this effect is even more pronounced at $4 \mathrm{kGy} / \mathrm{h}$ as indicated by the $2 \mathrm{GPa}$ value point at $3 \mathrm{MGy}$.

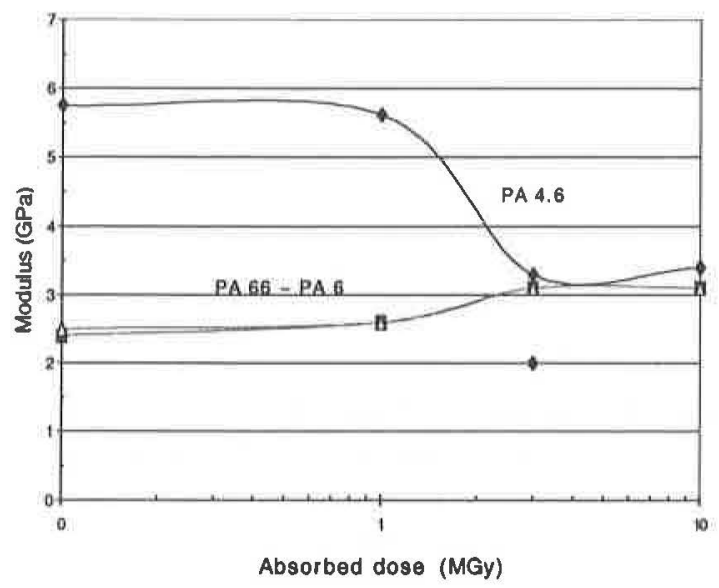

Fig. 4 -Comparison of the radiation degradations of 3 different polyamides.

If the materials are to be used for other properties such as translucence for example, the phenomena to be taken into account are completely different, and the dose limits are much lower. Usually, very strong synergistic effects between radiation and other parameters are observed in organic optical materials such as scintillators and optical fibres $[22,23]$.

For fluorocarbon cooling fluids, a special care must be paid to alcanes and hydrofluoroalcanes, which are usually present as impurities, and of which the $\mathrm{C}-\mathrm{H}$ bonds content opens the way to the reactive hydrofluoric acid evolution during the radiolytic process. It has been shown that HF may be eliminated by means of active filters in the cooling circuit, allowing these fluids to be used up to several tens of $\mathrm{kGy}$. The reduction of optical transmission can be used as an ageing-monitoring technique. It has also been checked that the activation of fluoride remains below acceptable level. The tri-fluoro-iodo-methane $\left(\mathrm{CF}_{3} \mathrm{I}\right)$ has proven to be very sensitive to radiation and cannot be used for the cooling of the inner tracker of LHC experiments.

\section{CONCLUSION}

At CERN, for almost 40 years, emphasis has been put on the proper selection of insulators to be used in the radiation environment around particle accelerators. A particular attention has been brought on the dose-rate effect study. Other ageing parameters have been identified; they have a synergistic effect with radiation. From our experience, it is possible to have a fair assessment of the lifetime of a component in a given radiation environment. Further studies are still ongoing about optical and luminescent materials and about organic cooling fluids.

\section{REFERENCES}

[1] H. Schönbacher and F. Coninckx: Doses to the CERN 450-GeV Super Proton Synchrotron and estimate of radiation damage.

Nuc. Instr. \& Meth. A 288, (1990)

[2] IEC 544: Guide for determining the effects of ionising radiation on insulating materials, Part I: radiation interaction, Part II: procedures for irradiation and tests, Part IV: classification system for service in radiation environments.

[3] H. Schönbacher et al.: Compilation of Radiation Damage Test Results, CERN Reports 79-04 (1979), 79-08 (1979), 81-05 (1981), 82-10 (1982), 85-02 (1985), 89-12 (1989) and 98-01 (1998) (CERN)

[4] D. W. Clegg and A. A. Collyer: Irradiation effects on polymers. Elsevier Science Publishers Ltd, London, 1991

[5] A. Chapiro et al.: What is ageing ? Are there still problems to be solved? Nuc. Instr. \& Meth. B 131, pp x-xii (1997)

[6] OECD, Nuclear Safety : Survey on organic components in NPPs. NEA/CSNI/R(98)7 (1999)

[7] G. A. Patfoort : Polymers.

E. Story_Scientia, Gent, 1974

[8] A. Tourelle : Methods of aging diagnostics and estimate of life time. Proceedings of "Aging and life time of polymers used in electrical and electronic industries", Montpellier, 1995

[9] F. Schue and A. Collet : Chemical aging of polymers. Same proceedings as [8] 
[10] K. T. Gillen and R. L. Clough : Techniques for monitoring heterogeneous oxidation of polymers. Handbook of Polymer Science and Technology, Vol. 2, 167-202, Marcel Dekker Inc, 1989

[11] T.A. Shook and J.B. Gardner : Cable Indenter Aging Monitor. Franklin Research Center, EPRI Report NP-5920 (1988)

[12] Long-term aging and Loss-of-Coolant Accident (LOCA); Electrical cables. NUREG/CR-6202 + IPSN 94-03 (1994)

[13] B. Nicquevert and C. Hauviller (eds) : Proceedings of the first International Workshop on Advanced Materials for High Precision Detectors. CERN 94-07 (1994)

[14] M. Tavlet and L. Hominal : Shear tests on adhesives for magnet collars for the LHC. Cryogenics Vol. 38, No 1, pp 47-50 (1998)

[15] P. Maier and A. Stolarz : Long-term radiation effects on commercial cableinsulating materials irradiated at CERN. CERN 83-08 (1983)

[16] M. Tavlet et al.: Assessment of radiation damage to a halogen-free cable jacket. ACS Symposium Series No 475, 1991

[17] K. T. Gillen and R. L. Clough : A kinetic model for predicting oxidative degradation rates in combined radiation-thermal environments. Journal of Polymer Sciences, Vol. 23 (1985)
[18] F. Hanisch et al.: Effects of radiation types and dose rates on selected cable-insulating materials. Radiat. Phys. Chem. Vol. 30, No 1, pp 1-9, 1987

[19] S. M. Spießberger et al.: Reactor irradiation effects on the ultimate tensile and the interlaminar shear strength of carbon fibre reinforced epoxies at $77 \mathrm{~K}$.

Cryogenics Vol. 38, No 1, pp 79-84 (1998)

[20]H. Schönbacher et al : Radiation tests at cryogenic temperature on selected organic materials for LHC. CERN 96-05 (1996)

[21]S. G. Burnay : Effect of pre-stressing on the behaviour of CFRP under gamma irradiation: ESA Journal 1992, Vol. 16

[22] K. Wick et al.: Recovery and dose rate dependence of radiation damage in scintillators, wavelength shifters and light guides. Nuc. Instr. \& Meth. B 61, (1991)

[23] H. Henschel, O. Köhn and H. U. Schmidt : Influence of dose rate on radiation induced loss in optical fibres. SPIE Vol. 1399 (1990) 